\title{
Examination of fund age and size and its impact on hedge fund performance
}

\section{Meredith Jones}

PerTrac Financial Solutions, 58 West 40th Street, 4th Floor, New York, NY 10018, USA.

Tel: +1 (615) 297 2500; Fax: +1 (615) 297 4499; E-mail: meredith.jones@pertrac.com

Received (in revised form): 7th September, 2006

Meredith Jones is Managing Director, PerTrac Financial Solutions, New York.

\section{Practical applications}

Hedge funds are an increasingly popular investment option for both high net worth and institutional investors. With an increasing number of funds from which to choose, it is imperative that investors find ways to narrow down their investment options and evaluate managers.

\section{Abstract}

This paper attempts to discover whether smaller, younger hedge funds offer stronger performance than larger, older hedge funds. Using indices created with six subsets of hedge fund data (small, medium, large, young, mid-age and older funds, as defined herein) and Monte Carlo simulations, we examine the performance, volatility and risk profiles of each fund group.

Derivatives Use, Trading \& Regulation (2007) 12, 342-350. doi:10.1057/palgrave.dutr.1850052

Keywords: hedge funds; age; size; performance; returns; risk

\section{INTRODUCTION}

There is an old adage that states 'Age and treachery will always overcome youth and skill'. However, it is questionable whether or not this maxim is indeed true when it comes to hedge fund investments. The issue of hedge fund age and size, and its potential impact on performance has been a topic of frequent debate over the past five years. Certainly, hedge funds - large and small, or new and old - have experienced their fair share of both positive and negative publicity. For example, Julian Robertson's Tiger Funds drew attention to the potential pitfalls surrounding large, established funds, when after decades of successful performance, he shut down his flagship fund due in large part to the difficulties he encountered in managing his massive fund. On the other hand, smaller, younger funds like Integral Capital Management have made headlines for both operational and performance woes. This paper will attempt to examine the performance of hedge funds, classified into six size and age subcategories, to determine if any one group has a performance advantage over the others.

\section{SUPERSIZE ME?}

In many areas, bigger is perceived as better. However, in hedge funds, the opposite seems
Derivatives Use, Trading \& Regulation, Vol. 12 No. 4, 2007 pp. $342-350$ (C) 2007 Palgrave Macmillan Ltd 1357-0927 \$30.00 
to be the case. We created three size-based hedge fund indices by first combining the hedge fund performance records from the Hedge Fund Research, HedgeFund.net, Altvest from InvestorForce and Barclays Global HedgeSource databases into a single 'master' database.

Duplicate hedge fund records as well as records for funds of funds were removed. Reports were then run to find the monthly return and monthly fund size for each fund from January 1996 to July 2006. Based on its then-current fund size, all funds were recategorised each month and divided into three classes: funds with less than or equal to $\$ 100 \mathrm{~m}$ under management; funds with over $\$ 100 \mathrm{~m}$ up to $\$ 500 \mathrm{~m}$ under management; and funds with over $\$ 500 \mathrm{~m}$. A simple mean of all monthly returns in each of the three categories was calculated for each month. If a fund did not have a reported fund size in a given month, it was not included in any of the size-based indices for that month. Funds denominated in a non-US currency, with a very different value from the US dollar (USD) (eg JPY, CHF, NOK and SEK), were also excluded from the size-based indices, as it was not clear whether the monthly fund sizes reported for such funds were denominated in USD or in the native currency, making accurate size categorisation problematic. The sample of funds included in each of the three indices varied from month to month. On average, the small-sized index contained, 1,790.5 funds per month, whereas the medium-sized and large-sized indices contained 479.9 and 136.5 funds per month, respectively. In all three cases, the earlier monthly samples contained less funds than later samples. The three size-based indices that were created using this information are shown below in Figure 1.

As the summary risk-reward tables below show, small funds clearly provide the best investment option, given that 'best' is simply defined as providing maximised returns. For example, the small funds index in our study provided an annualised return of 15.46 per cent while maintaining an annualised standard deviation of 6.31 per cent and annualised downside deviation of 2.58 per cent. In comparison, medium-sized funds produced an annualised return of 12.50 per cent with slightly lower annualised standard and downside deviations of 5.89 and 2.10 per cent, respectively. The large fund index in the study produced the lowest annualised return of 11.93 per cent and also the lowest annualised standard deviation at 5.72 per cent. Its annualised

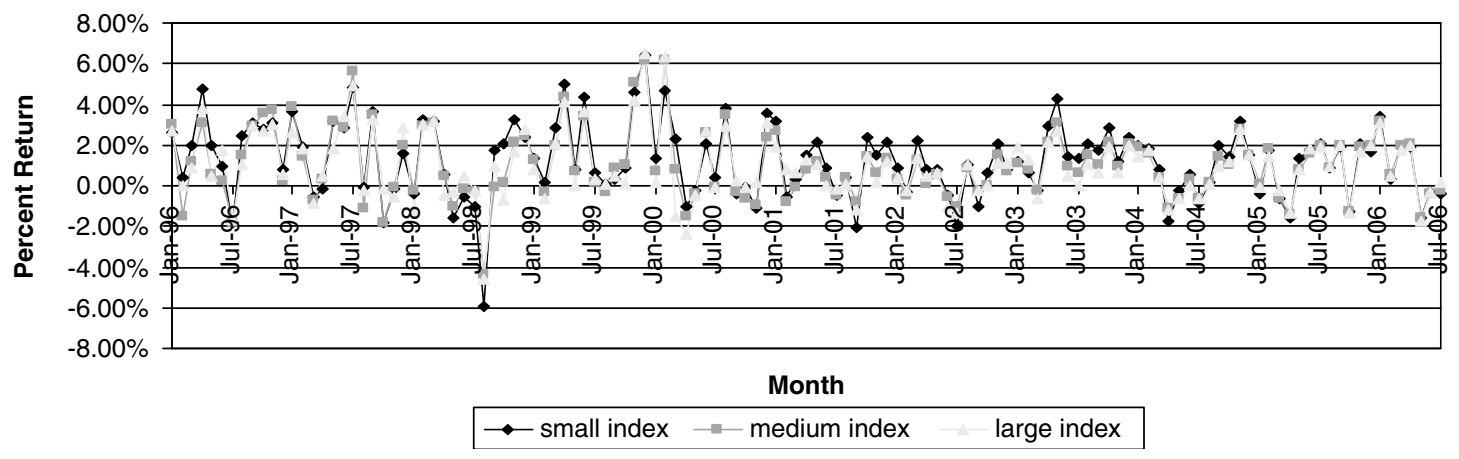

Figure 1: Performance of small, medium and large hedge funds 
downside deviation of 2.16 per cent falls in the middle of the three groups, although it is very similar to that of the medium-sized fund index in the study (Tables 1-3).

The pattern of smaller funds outperforming larger funds is repeated when examining Monte Carlo simulations performed on the created indices. The simulations were run five years forward, based on the full historical data of each index (January 1996 to July 2006), using the S\&P 500 Index (Total Return) as the market benchmark, and a 2 per cent annualised risk-free rate of return and minimum acceptable return (MAR). Using the Bootstrap method, we ran 1,000 simulations with quarterly rebalancing, which provided a minimum and maximum predicted return for each index. As shown below, the maximum simulated return for the large hedge fund index was 21.57 per cent, while

Table 1: Small HF index (up to $\$ 100 m$ )

\begin{tabular}{lccl}
\hline Risk table & Month & Quarter & Annualised \\
\hline Compound return & $1.20 \%$ & $3.66 \%$ & $15.46 \%$ \\
Arithmetic mean & $1.22 \%$ & $3.76 \%$ & $\mathrm{~N} / \mathrm{A}$ \\
Standard deviation & $1.82 \%$ & $3.71 \%$ & $6.31 \%$ \\
Semi deviation & $1.87 \%$ & $3.92 \%$ & $6.49 \%$ \\
Gain deviation & $1.30 \%$ & $2.90 \%$ & $4.50 \%$ \\
Loss deviation & $1.08 \%$ & $1.70 \%$ & $3.72 \%$ \\
Down deviation (5.00\%) & $0.90 \%$ & $1.41 \%$ & $3.13 \%$ \\
Down deviation (2.00\%) & $0.81 \%$ & $1.15 \%$ & $2.79 \%$ \\
Down deviation (0\%) & $0.75 \%$ & $0.98 \%$ & $2.58 \%$ \\
Sharpe (2.00\%) & 0.58 & 0.88 & 2.01 \\
Sortino (5.00\%) & 0.88 & 1.73 & 3.06 \\
Sortino (2.00\%) & 1.29 & 2.76 & 4.47 \\
Sortino (0\%) & 1.62 & 3.72 & 5.6 \\
Skewness & -0.2 & -0.08 & \\
Kurtosis & 1.19 & -0.09 & \\
\hline
\end{tabular}

Table 2: Medium HF index lover $\$ 100 m$ up to $\$ 500 m$ )

\begin{tabular}{llll}
\hline Risk table & Month & Quarter & Annualised \\
\hline Compound return & $0.99 \%$ & $2.99 \%$ & $12.50 \%$ \\
Arithmetic mean & $1.00 \%$ & $3.07 \%$ & $\mathrm{~N} / \mathrm{A}$ \\
Standard deviation & $1.70 \%$ & $3.26 \%$ & $5.89 \%$ \\
Semi deviation & $1.50 \%$ & $2.93 \%$ & $5.20 \%$ \\
Gain deviation & $1.37 \%$ & $2.84 \%$ & $4.76 \%$ \\
Loss deviation & $0.78 \%$ & $1.71 \%$ & $2.70 \%$ \\
Down deviation (5.00\%) & $0.78 \%$ & $1.18 \%$ & $2.72 \%$ \\
Down deviation (2.00\%) & $0.67 \%$ & $0.93 \%$ & $2.33 \%$ \\
Down deviation (0\%) & $0.61 \%$ & $0.79 \%$ & $2.10 \%$ \\
Sharpe (2.00\%) & 0.49 & 0.79 & 1.7 \\
Sortino (5.00\%) & 0.74 & 1.49 & 2.56 \\
Sortino (2.00\%) & 1.22 & 2.69 & 4.22 \\
Sortino (0\%) & 1.63 & 3.8 & 5.64 \\
Skewness & 0.49 & 0.48 & \\
Kurtosis & 1 & 0.9 & \\
\hline
\end{tabular}

Table 3: Large HF index (over $\$ 500 m$ )

\begin{tabular}{llll}
\hline Risk table & Month & Quarter & Annualised \\
\hline Compound return & $0.94 \%$ & $2.86 \%$ & $11.93 \%$ \\
Arithmetic mean & $0.96 \%$ & $2.92 \%$ & $\mathrm{~N} / \mathrm{A}$ \\
Standard deviation & $1.65 \%$ & $2.88 \%$ & $5.72 \%$ \\
Semi deviation & $1.48 \%$ & $2.74 \%$ & $5.14 \%$ \\
Gain deviation & $1.35 \%$ & $2.37 \%$ & $4.66 \%$ \\
Loss deviation & $0.88 \%$ & $1.35 \%$ & $3.04 \%$ \\
Down deviation (5.00\%) & $0.79 \%$ & $1.12 \%$ & $2.75 \%$ \\
Down deviation (2.00\%) & $0.69 \%$ & $0.85 \%$ & $2.38 \%$ \\
Down deviation (0\%) & $0.62 \%$ & $0.69 \%$ & $2.16 \%$ \\
Sharpe (2.00\%) & 0.48 & 0.84 & 1.66 \\
Sortino (5.00\%) & 0.68 & 1.46 & 2.34 \\
Sortino (2.00\%) & 1.13 & 2.79 & 3.92 \\
Sortino (0\%) & 1.51 & 4.12 & 5.23 \\
Skewness & 0.43 & 0.29 & \\
Kurtosis & 1.48 & 0.65 & \\
\hline
\end{tabular}


the medium hedge fund index showed a maximum simulated return of 23.57 per cent, and the small hedge fund index produced a maximum simulated return of 27.49 per cent. The mean simulated annualised returns followed the same pattern: best for the small index, worst for the large index (Tables 4-6).

However, while the small fund index in the study produced, and had the potential to produce in the future, a higher annualised return, it also had a higher volatility profile than did the larger- and medium-sized indices. In Tables 1, 2 and 3 above, we have already noted that the smaller funds had the highest standard and downside deviation of the three fund

Table 4: Small fund index Monte Carlo simulation - annualised return

\begin{tabular}{ll}
\hline All porffolio statistics & Annualised return \\
\hline Number of simulations & 1,000 \\
Mean & $15.44 \%$ \\
Median & $15.43 \%$ \\
Standard deviation & $3.14 \%$ \\
Maximum & $27.49 \%$ \\
Minimum & $5.11 \%$ \\
\hline
\end{tabular}

Table 5: Medium fund index Monte Carlo simulation - annualised return

\begin{tabular}{ll}
\hline All portfolio statistics & Annualised return \\
\hline Number of simulations & 1,000 \\
Mean & $12.51 \%$ \\
Median & $12.44 \%$ \\
Standard deviation & $2.85 \%$ \\
Maximum & $23.57 \%$ \\
Minimum & $4.16 \%$ \\
\hline
\end{tabular}

Table 6: Large fund index Monte Carlo simulation - annualised return

\begin{tabular}{ll}
\hline All Portfolio statistics & Annualised return \\
\hline Number of simulations & 1,000 \\
Mean & $11.94 \%$ \\
Median & $11.91 \%$ \\
Standard deviation & $2.74 \%$ \\
Maximum & $21.57 \%$ \\
Minimum & $4.74 \%$ \\
\hline
\end{tabular}

Table 7: Small fund index Monte Carlo simulation - drawdown

\begin{tabular}{ll}
\hline All portfolio statistics & Maximum drawdown \\
\hline Number of simulations & 1,000 \\
Mean & $-4.49 \%$ \\
Median & $-3.80 \%$ \\
Standard deviation & $2.26 \%$ \\
Maximum & $-14.23 \%$ \\
Minimum & $-0.59 \%$ \\
\hline
\end{tabular}

groups. Using the same Monte Carlo simulation methods as above, we can extrapolate potential maximum drawdowns for the three indices. The maximum simulated drawdown of the small fund index is -14.23 per cent, while the medium-sized fund index came in significantly lower at -11.02 per cent, and the large fund index fell in the middle with a maximum simulated drawdown of -12.30 per cent (Tables 7-9).

The question therefore arises, why is performance higher for smaller funds? A number of factors may be responsible for the higher performance. First, smaller funds, because they have less capital to invest, can select only from their best investment ideas. Their positions are 
Table 8: Medium fund index Monte Carlo simulation - drawdown

\begin{tabular}{ll}
\hline All portfolio statistics & Maximum drawdown \\
\hline Number of simulations & 1,000 \\
Mean & $-3.77 \%$ \\
Median & $-3.57 \%$ \\
Standard deviation & $1.68 \%$ \\
Maximum & $-11.02 \%$ \\
Minimum & $-0.92 \%$ \\
\hline
\end{tabular}

Table 9: Large fund index Monte Carlo simulation - drawdown

\begin{tabular}{ll}
\hline All portfolio statistics & Maximum drawdown \\
\hline Number of simulations & 1,000 \\
Mean & $-3.84 \%$ \\
Median & $-3.54 \%$ \\
Standard deviation & $1.79 \%$ \\
Maximum & $-12.30 \%$ \\
Minimum & $-0.66 \%$ \\
\hline
\end{tabular}

generally smaller, so they can move more nimbly and attract less attention to their strategic moves than can large funds. Smaller funds may also be able to exploit small market inefficiencies and opportunities that larger funds may have to ignore. In the mean time, as assets increase, managers may be forced to change the strategy that made them initially successful by resorting to one of the following tactics to keep capital in play:

- Look outside the manager's area of expertise for additional investment opportunities.

- Concentrate more money into top tier investment ideas.
- Move from top investment ideas to second, third or fourth tier opportunities.

- Keep a large amount of the funds in cash, thereby lowering the returns.

- Farm out portions of the investment management process to other sub-advisors who may not have the manager's level of experience, credentials or back office infrastructure and

- Spend more time managing the organisation and less time managing the funds, leaving the trading in the hands of less-seasoned personnel.

Additionally, a considerable portion of large funds' assets may be from institutional investors, such as pension funds, prompting large fund managers to invest more conservatively than small fund managers, who mainly handle the assets of less risk-averse, high net worth individuals.

Of course, there are disadvantages to investing with small funds as well. Our small fund index exhibited the highest volatility of the three. Fund infrastructure may be weak, and operational problems can plague or even destroy a fund. The fund assets may be concentrated with a few limited partners, which can cause a cascading effect if just one or two investors redeem, and the manager has to liquidate positions prematurely. Finally, smaller fund managers may take more risks owing to a desire to establish a strong track record and attract additional assets, or because of a lack of established risk-control measures. ${ }^{1}$ Meanwhile, larger funds will often have a more well-developed infrastructure and back office, well-defined risk-control measures, steady performance that caters to their institutional limited partners and a diversified investor-base. ${ }^{2,3}$ To choose the best fund, it is 
therefore critical that small, medium and large funds be fully evaluated before an investment is made.

\section{YOUTH OVER EXPERIENCE?}

Another popular subject of debate is whether hedge funds really do produce their best returns early in their history, regardless of assets under management. To try to shed light on this issue, three age-based indices from the same master hedge fund database referred to above, were created from the Hedge Fund Research, HedgeFund.net, Altvest from InvestorForce and Barclays Global HedgeSource databases. Reports were then run to find the monthly return for each fund from January 1996 to July 2006. Based on its then-current fund age all funds were recategorised each month and divided into three classes: funds with less than a two-year track record, funds with two to four years of performance and funds with more than four years of performance. A simple mean of all monthly returns in each of the three categories was calculated for each month. The indices that were created using this information are shown below in Figure 2.
Like the smallest funds, the youngest funds in this study were the strongest performers. The young fund index in the study produced an annualised return of 17.50 per cent, while maintaining an annualised standard deviation of 5.97 per cent and an annualised downside deviation of 2.24 per cent. In comparison, the mid-age fund index produced an annualised return of 14.10 per cent, with annualised standard and downside deviations of 6.39 and 2.81 per cent, respectively. The index composed of oldest funds in the study produced the lowest annualised return of 11.84 per cent and also the lowest annualised standard deviation, of 6.32 per cent. Its downside deviation of 2.85 per cent falls in the middle of the three groups, although, again, it is so close to that of the mid-age index that the difference may not be significant (Tables 10-12).

The pattern of younger funds outperforming older funds is repeated when examining Monte Carlo simulations performed on the created indices. As with the size-based indices, the simulations were run five years forward, based on the full history of the indices from January 1996 to July 2006. The S\&P 500 Index (Total Return) was again used as the market benchmark, with a

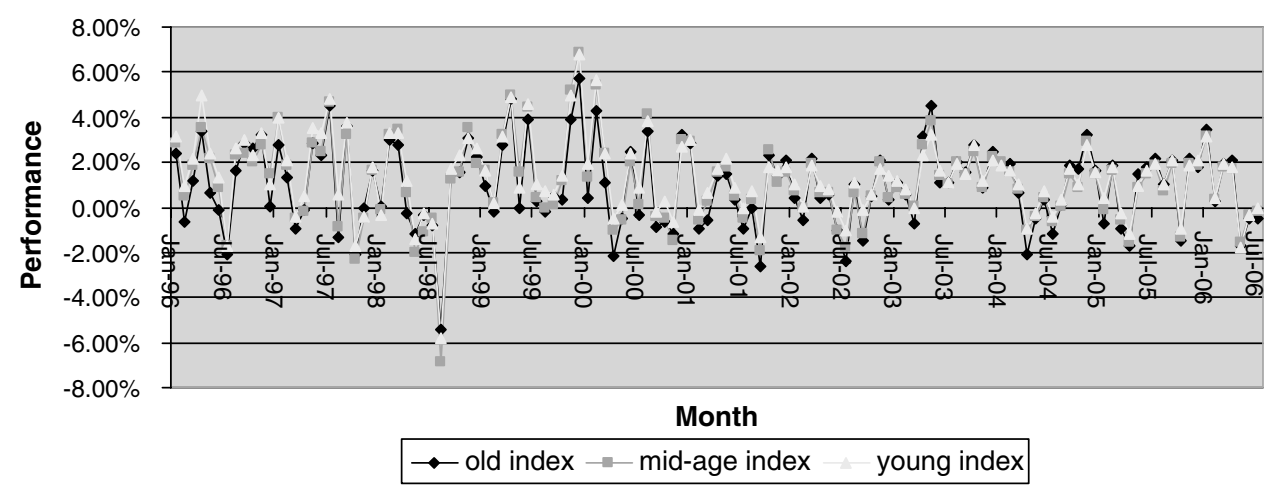

Figure 2: Monthly performance of various age hedge funds 
Table 10: Young hedge fund index (up to $2 \mathrm{Yr}$ )

\begin{tabular}{llll}
\hline Risk table & Month & Quarter & Annualised \\
\hline Compound return & $1.35 \%$ & $4.11 \%$ & $17.50 \%$ \\
Arithmetic mean & $1.37 \%$ & $4.21 \%$ & $\mathrm{~N} / \mathrm{A}$ \\
Standard deviation & $1.72 \%$ & $3.63 \%$ & $5.97 \%$ \\
Semi deviation & $1.73 \%$ & $3.70 \%$ & $5.99 \%$ \\
Gain deviation & $1.31 \%$ & $2.92 \%$ & $4.55 \%$ \\
Loss deviation & $1.13 \%$ & $1.70 \%$ & $3.92 \%$ \\
Down deviation (5.00\%) & $0.78 \%$ & $1.13 \%$ & $2.70 \%$ \\
Down deviation (2.00\%) & $0.70 \%$ & $0.90 \%$ & $2.41 \%$ \\
Down deviation (0\%) & $0.65 \%$ & $0.78 \%$ & $2.24 \%$ \\
Sharpe (2.00\%) & 0.7 & 1.02 & 2.42 \\
Sortino (5.00\%) & 1.21 & 2.55 & 4.21 \\
Sortino (2.00\%) & 1.71 & 4 & 5.91 \\
Sortino (0\%) & 2.09 & 5.31 & 7.24 \\
Skewness & -0.11 & 0.15 & \\
Kurtosis & 2.18 & 0.45 & \\
\hline
\end{tabular}

2 per cent annualised risk-free rate of return and MAR. Using the Bootstrap method, we ran 1,000 simulations with quarterly rebalancing, which provided a minimum and maximum simulated return for each index. As shown below, the maximum simulated return for the old hedge fund index was 23.35 per cent, while the mid-age hedge fund index showed a maximum simulated return of 25.70 per cent, and the young hedge fund index produced a maximum simulated return of 27.62 per cent. Mean simulated returns followed the same pattern (Tables 13-15).

However, unlike the smallest funds in the study, which exhibited the highest historical and simulated future returns, as well as the highest historical and simulated future volatility and drawdowns among the three size-based indices,
Table 11: Mid-age hedge fund index (2-4 Years)

\begin{tabular}{llll}
\hline Risk table & Month & Quarter & Annualised \\
\hline Compound return & $1.11 \%$ & $3.35 \%$ & $14.10 \%$ \\
Arithmetic mean & $1.12 \%$ & $3.46 \%$ & $\mathrm{~N} / \mathrm{A}$ \\
Standard deviation & $1.84 \%$ & $3.89 \%$ & $6.39 \%$ \\
Semi deviation & $1.87 \%$ & $4.01 \%$ & $6.48 \%$ \\
Gain deviation & $1.34 \%$ & $3.11 \%$ & $4.64 \%$ \\
Loss deviation & $1.20 \%$ & $1.93 \%$ & $4.15 \%$ \\
Down deviation (5.00\%) & $0.97 \%$ & $1.59 \%$ & $3.35 \%$ \\
Down deviation (2.00\%) & $0.87 \%$ & $1.32 \%$ & $3.02 \%$ \\
Down deviation (0\%) & $0.81 \%$ & $1.15 \%$ & $2.81 \%$ \\
Sharpe (2.00\%) & 0.52 & 0.76 & 1.8 \\
Sortino (5.00\%) & 0.72 & 1.33 & 2.5 \\
Sortino (2.00\%) & 1.08 & 2.17 & 3.74 \\
Sortino (0\%) & 1.36 & 2.9 & 4.71 \\
Skewness & -0.21 & 0.13 & \\
Kurtosis & 2.36 & 0.61 & \\
\hline
\end{tabular}

Table 12: Old hedge fund index

\begin{tabular}{llll}
\hline Risk table & Month & Quarter & Annualised \\
\hline Compound return & $0.94 \%$ & $2.84 \%$ & $11.84 \%$ \\
Arithmetic mean & $0.95 \%$ & $2.93 \%$ & $\mathrm{~N} / \mathrm{A}$ \\
Standard deviation & $1.83 \%$ & $3.50 \%$ & $6.32 \%$ \\
Semi deviation & $1.88 \%$ & $3.58 \%$ & $6.50 \%$ \\
Gain deviation & $1.24 \%$ & $2.63 \%$ & $4.29 \%$ \\
Loss deviation & $0.98 \%$ & $1.72 \%$ & $3.40 \%$ \\
Down deviation (5.00\%) & $1.01 \%$ & $1.60 \%$ & $3.49 \%$ \\
Down deviation (2.00\%) & $0.89 \%$ & $1.30 \%$ & $3.10 \%$ \\
Down deviation (0\%) & $0.82 \%$ & $1.12 \%$ & $2.85 \%$ \\
Sharpe (2.00\%) & 0.43 & 0.7 & 1.5 \\
Sortino (5.00\%) & 0.53 & 1 & 1.82 \\
Sortino (2.00\%) & 0.86 & 1.8 & 2.99 \\
Sortino (0\%) & 1.14 & 2.53 & 3.95 \\
Skewness & -0.14 & -0.09 & \\
Kurtosis & 0.32 & -0.37 & \\
\hline
\end{tabular}


Table 13: Young fund index Monte Carlo simulation -annualised return

\begin{tabular}{ll}
\hline All portfolio statistics & Annualised return \\
\hline Number of simulations & 1,000 \\
Mean & $17.50 \%$ \\
Median & $17.48 \%$ \\
Standard deviation & $3.01 \%$ \\
Maximum & $27.62 \%$ \\
Minimum & $7.80 \%$ \\
\hline
\end{tabular}

Table 14: Mid-age fund index Monte Carlo simulation - annualised return

\begin{tabular}{ll}
\hline All portfolio statistics & Annualised return \\
\hline Number of simulations & 1,000 \\
Mean & $14.10 \%$ \\
Median & $14.11 \%$ \\
Standard deviation & $3.16 \%$ \\
Maximum & $25.70 \%$ \\
Minimum & $4.23 \%$ \\
\hline
\end{tabular}

Table 15: Old fund index Monte Carlo simulation - annualised return

\begin{tabular}{ll}
\hline All portfolio statistics & Annualised return \\
\hline Number of simulations & 1,000 \\
Mean & $11.82 \%$ \\
Median & $11.81 \%$ \\
Standard deviation & $3.08 \%$ \\
Maximum & $23.35 \%$ \\
Minimum & $2.18 \%$ \\
\hline
\end{tabular}

the young fund index maintained the highest returns, without a corresponding impact on simulated future drawdowns. In fact, it is the
Table 16: Young fund index Monte Carlo simulation - drawdown

\begin{tabular}{ll}
\hline All portfolio statistics & Maximum drawdown \\
\hline Number of simulations & 1,000 \\
Mean & $-3.74 \%$ \\
Median & $-2.75 \%$ \\
Standard deviation & $2.28 \%$ \\
Maximum & $-13.04 \%$ \\
Minimum & $-0.53 \%$ \\
\hline
\end{tabular}

Table 17: Mid-age fund index Monte Carlo simulation - drawdown

\begin{tabular}{ll}
\hline All portfolio statistics & Maximum drawdown \\
\hline Number of simulations & 1,000 \\
Mean & $-4.95 \%$ \\
Median & $-3.92 \%$ \\
Standard deviation & $2.72 \%$ \\
Maximum & $-17.46 \%$ \\
Minimum & $-1.00 \%$ \\
\hline
\end{tabular}

Table 18: Old fund index Monte Carlo simulation - drawdown

\begin{tabular}{ll}
\hline All portfolio statistics & Maximum drawdown \\
\hline Number of simulations & 1,000 \\
Mean & $-5.28 \%$ \\
Median & $-5.15 \%$ \\
Standard deviation & $2.19 \%$ \\
Maximum & $-14.84 \%$ \\
Minimum & $-1.45 \%$ \\
\hline
\end{tabular}

mid-age hedge fund index that had the highest simulated maximum drawdown of the group, at least suggesting that those funds exhibit 
somewhat of a 'sophomore slump', before moving towards the more institutional profile of their older peers. Using the same Monte Carlo simulation methods as above, we can extrapolate potential maximum drawdowns for each group of funds. The maximum simulated maximum drawdown of the young fund index is -13.04 per cent, while the old fund index came in at -14.84 per cent, and the mid-age fund group posted a maximum simulated maximum drawdown of -17.46 per cent (Tables 16-18).

\section{CONCLUSION}

While performance is certainly not the only metric by which to select a hedge fund investment, an analysis of hedge fund performance for small, medium and large hedge funds, as well as of young, mid-age and older funds, suggests that investors who wish to maximise return should start their search by looking for younger, smaller funds. Investors who wish to maximise capital preservation should begin their hedge fund screening with larger, older funds. However, it is always important to consider the qualitative aspects of any fund, in addition to historical and simulated future returns, before making an investment.

\section{References}

1 Gregoriou, G. N. (2002) 'Hedge Fund Survival Lifetimes', Journal of Asset Management, Vol. 3, No. 3, pp. 237-252.

2 Amman, E. and Moerth, P. (2005) 'Impact of Hedge Fund Size on Hedge Fund Performance', Working Paper Series in Finance No.11, University of St. Gallen, Switzerland.

3 Gregoriou, G. N. and Rouah, F. (2003) 'Large versus Small Hedge Funds: Does Size Affect Performance', Journal of Alternative Investments, Vol. 5, No. 5, pp. 75-77. 